\title{
Toxicidad y afectación en la locomoción de Triatoma dimidiata (Latreille 1811) (Hemiptera: Reduviidae: Triatominae) tratados con análogos de girgensohnina
}

\author{
Toxicity and affectation in locomotion of Triatoma \\ dimidiata (Latreille 1811) (Hemiptera: Reduviidae: \\ Triatominae) treated with analogues of girgensohnine
}

Edwin-R Escobar ${ }^{1}$; Aurora-L Carreño-Otero'; Vladimir-V Kouznetsov' ${ }^{1}$;onny-E Duque ${ }^{1}$

Forma de citar: Escobar E, Carreño-Otero AL, Kouznetsov VV, Duque JE. Toxicidad y afectación en la locomoción de Triatoma dimidiata (Latreille 1811) (Hemiptera: Reduviidae: Triatominae) tratados con análogos de girgensohnina. Rev Univ Ind Santander Salud. 2018; 50(3): 205-213. doi: http://dx.doi.org/10.18273/revsal.v50n3-2018005 C) (i)

\section{Resumen}

Introducción: Análogos del alcaloide girgensohnina, diseñados y sintetizados para inhibir a la enzima acetilcolinesterasa, han presentado efecto insecticida sobre insectos vectores de enfermedades. Objetivo: Determinar la actividad insecticida de 12 análogos sintéticos de gingersohnina sobre ninfas del primer estadio de Triatoma dimidiata. Materiales y Métodos: Se tomó como referencia el protocolo de la OMS 2005. Ninfas del primer estadio de $T$. dimidiata fueron expuestas a los análogos por aplicación tópica y exposición a superficies para determinar las dosis letales $\left(\mathrm{DL}_{50}\right.$ y $\left.\mathrm{DL}_{95}\right)$ y verificar alteración en la locomoción mediante el software Videomex V. Resultados: El análogo 6 presentó la mayor actividad insecticida a las $72 \mathrm{~h}$ con una mortalidad del 20,8\% $\pm 3,57$ para el tratamiento de topicación $25 \% \pm 0,00$ para las superficies no porosas. Las dosis letales de la molécula 6 fueron: $\mathrm{DL}_{50} 1036,8 \pm$ $0,38 \mathrm{ng} /$ insecto y $\mathrm{DL}_{95} 3991,4 \pm 0,50 \mathrm{ng} /$ insecto respectivamente. Con respecto a la locomoción, el análogo indujo un comportamiento similar al insecticida comercial deltametrina.

Palabras clave: Triatoma dimidiata; Enfermedad de Chagas; Análogos sintéticos de girgensohnina; Videomex V.

\begin{abstract}
Introduction: Analogs of the girgensohnine alkaloid, designed and synthesized to inhibit acetylcholinesterase enzyme, have presented an insecticidal effect on vectors insects of diseases. Objective: To determine the insecticidal activity of 12 synthetic analogs of gingersohnine in first stage nymphs of Triatoma dimidiata. Materials and methods: WHO protocol 2005, using exploratory doses, was used to determine the best molecule according to its mortality. First stage nymphs of $T$. dimidiata were exposed to different doses of the analogs by topical application and surface exposure. Lethal doses $\left(\mathrm{LD}_{50}\right.$ and $\left.\mathrm{DL}_{95}\right)$ and alterations in locomotion using Videomex $\mathrm{V}$ software were

1. Universidad Industrial de Santander, Bucaramanga, Santander, Colombia.

Correspondencia: Jonny E. Duque. Dirección: Km 2 Vía El Refugio, Guatiguara Piedecuesta. Teléfono: 5776344000 Ext 3503. Correo electrónico: jonedulu@uis.edu.co
\end{abstract}


determined. Results: Analog 6 presented the highest insecticidal activity at $72 \mathrm{~h}$ with a mortality of $20.8 \% \pm 3.57$ for topical treatment and $25 \% \pm 0,0$ for non-porous surfaces evaluation. Analog 6 lethal doses were $\mathrm{LD}_{50} 1036.8 \pm$ $0.38 \mathrm{ng} /$ insecto and $\mathrm{DL}_{95} 3991.4 \pm 0.508 \mathrm{ng} /$ insecto, respectively. With regard to locomotion, analogue 6 induced a similar behavior to that observed for commercial insecticide deltametrin.

Keywords: Triatoma dimidiata; Chagas disease; Synthetic analogues of girgensohnine; Videomex V.

\section{Introducción}

La enfermedad de Chagas o tripanosomiasis americana, descubierta en 1909 por el médico Carlos Ribeiro Justiniano Chagas, representa un grave problema de salud pública en Latinoamerica ${ }^{1}$. El agente etiológico de esta dolencia es el protozoario flagelado Tripanosoma cruzi el cual se transmite principalmente por el contacto con las heces de los insectos hematófagos denominados triatominos. Para la enfermedad de Chagas, se estima que alrededor del mundo hay ocho millones de personas infectadas con el parásito ${ }^{2}$. Aunque es endémica en Centro América y Sur América, con una incidencia de 42.500 casos y 21.000 muertes cada año, ya se registran casos de infección en el sur de Estados Unidos ${ }^{3}$. Medicamentos como el nirfutimox y benznidazol, eficientes en una etapa temprana de la patología, no han impactado considerablemente en la reducción de los casos debido a que en la mayoría de los pacientes esta patología se expresa de forma asintomática, por lo cual no son tratados en su etapa controlable 4 . Aunque existen diferentes formas de transmisión como por la transfusión de sangre, por vías orales, transplacentarías o por el canal de parto, accidentes de laboratorio y trasplante de órganos, la principal ocurre cuando el triatomino, después de alimentarse de su hospedero, defeca depositando formas infectivas del parasito sobre la piel, las cuales ingresan al cuerpo por heridas o la abrasión causada al rascarse ${ }^{1,5}$.

En la actualidad, existen alrededor de 151 especies de triatominos clasificados en cinco tribus y 15 géneros ${ }^{6}$ Triatominae (Hemiptera: Reduviidae. Las especies Triatoma infestan (Klug, 1834), Rhodnius proxilus (Stal, 1859) y Triatoma dimidiata (Latreille, 1811) son los principales vectores del continente Americano ${ }^{7}$. En Colombia existen 24 especies de triatominos, de los cuales 15 se encuentran infectados naturalmente por $T$. cruzi, siendo $R$. prolixus y $T$. dimidiata las principales especies transmisoras de la enfermedad. T. dimidiata se encuentra en los departamentos de Casanare, Cesar, Cundinamarca, Guajira, Magdalena, Santander, Norte de Santander, Tolima y Vichada ${ }^{8}$. Se ha establecido que la forma más efectiva para disminuir la incidencia de esta parasitemia es por medio de la acción conjunta de tres factores: el mejoramiento de las viviendas en las zonas rurales afectadas, la educación de las personas, sobre todo aquellos en sitios de riesgo, y el control del vector mediante el empleo de insecticidas. Este último método es considerado la mejor estrategia para reducir la incidencia de la enfermedad, debido a que la disminución del vector lleva a la interrupción el ciclo de transmisión? Entre los principales insecticidas comerciales se tienen a los compuestos organofosforados, carbamatos y piretroides 9 , sin embargo, debido su aplicación excesiva en las últimas décadas, se ha reportado resistencia principalmente a piretroides ${ }^{10,11}$.

En la búsqueda de moléculas con acción insecticida se han sintetizado nuevos análogos de la girgensohnina, alcaloide natural presente en la Girgensohnia oppositiflora, arbusto que crece en los desiertos de Rusia e Irán. Debido a que este metabolito mostró inhibir a la acetilcolinesterasa (AChE) de forma moderada, se generó una serie nueva de análogos buscando potenciar esta acción ${ }^{12}$. Las moléculas sintetizadas mostraron inhibir a la AChE mejor que el alcaloide natural y su evaluación sobre larvas de Aedes aegypti (Diptera: Culicidae) y ninfas de $R$. prolixus presentaron acción insecticida $\left(\mathrm{DL}_{50}\right.$ de $225.60 \mathrm{ng}$ /insecto y $\mathrm{LD}_{95} 955.90$ ng/insecto) con el análogo número ${ }^{13,14}$. Sin embargo, estas moléculas cuando se evaluaron sobre ninfas de un estado más avanzado del ciclo de vida como el quinto estadio su acción insecticida fue significativamente menor ${ }^{13}$. De acuerdo con esta baja acción insecticida en ninfas de quinto estadio, y aunque se cuenta con antecedentes de que estas moléculas inhiben la AChE en culícidos, características estructurales como la presencia de un grupo alfa ciano y arilos sustituidos en su estructura podrían indicar un modo de acción comportamental similar al de moléculas que afectan el sistema nervioso lo cual podría ser detectado por pruebas de locomoción que reflejen alteración en la actividad $^{15}$.

En este trabajo se propuso evaluar la actividad insecticida de 12 análogos del alcaloide girgensohnina sobre ninfas del primer estadio de $T$. dimidiata, siendo la primera vez que se evalúan sobre esta especie la cual 
es uno de los principales vectores de la enfermedad de Chagas en Colombia, y de esta manera determinar las dosis letales (DL) para los análogos con mayor acción insecticida y establecer si es afectada la locomoción de las ninfas como modelo de evaluación comportamental en respuesta a su exposición a estos análogos.

\section{Materiales y métodos}

\section{Material biológico}

Para los experimentos se utilizó la cepa de referencia de $T$. dimidiata CINTROP-UIS colonizada en laboratorio desde el 1 agosto de 1997 con ejemplares colectados del municipio de San Joaquín, Santander - Colombia. Ninfas y adultos de este insecto fueron alimentados cada ocho días con sangre de gallina Gallus gallus para obtener huevos y ninfas para los experimentos. Debido a que el ciclo de vida de esta especie desde huevo a adulto es de 269 días y puede oscilar entre 174 a 598 días $^{16}$, para los bioensayos se

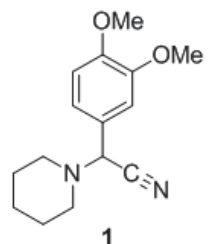

1<smiles>N#CC(c1ccc2c(c1)OCO2)N1CCCCC1</smiles>

$\underline{5}$<smiles>COc1cc(C(C#N)N2CCCCC2)cc(OC)c1OC</smiles>

$\underline{9}$

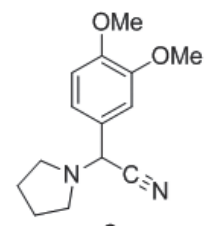

$\underline{2}$

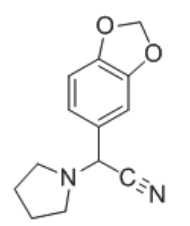

$\underline{6}$

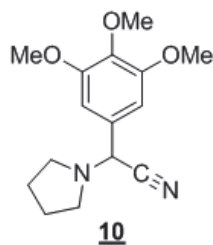

$\underline{10}$
Figura 1. Análogos evaluados del alcaloide girgensohnina. ${ }^{14}$

\section{Dosis Exploratorias (DE)}

El modelo experimental de este trabajo siguió los lineamientos de Cuadros, et al. para bioensayos con triatominos donde primero se inicia evaluando la acción insecticida con las DE para cada uno de los análogos ${ }^{13}$. Como DE se emplearon 50, 12,5, 2,5 ng/i, el control positivo fue deltametrina a las dosis $50 ; 0,7 ; 0,03 \mathrm{ng} / \mathrm{i}$, utilizaron únicamente ninfas de 24 a 36 horas de edad, las cuales estaban en ayuno desde la eclosión y con un peso promedio de $1,4 \pm 2,1 \mathrm{mg}$.

\section{Moléculas y material químico}

Los 12 análogos de girgensohnina fueron diseñados y generados empleando el protocolo sintético reportado por Carreño-Otero, et al. ${ }^{14}$ Tanto el alcaloide natural como sus análogos estructurales se clasifican como a-aminonitrilos constituidos por anillos $\mathrm{N}$-heterocíclicos de piperidina, pirrolidina, morfolina y N-metilpiperacina, los cuales son fragmentos ampliamente encontrados en metabolitos secundarios de las plantas. Las estructuras de los análogos 1-12 evaluados se presentan en la Figura 1, como control positivo de mortalidad se utilizó el insecticida de grado técnico deltametrina (99,5\%-ChemService), piretroide utilizado para el control vectorial en Santander. Para todas las diluciones y controles negativos se utilizó acetona $\left(99,98 \%\right.$ Merck $\left.^{\hat{a}}\right)$ como disolvente.
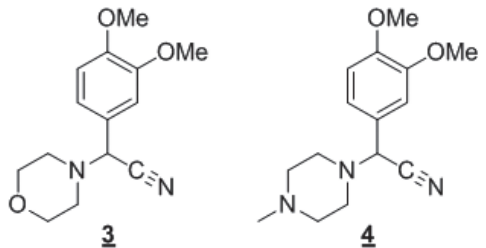<smiles>N#CC(c1ccc2c(c1)OCO2)N1CCOCC1</smiles><smiles>CN1CCN(C(C#N)c2ccc3c(c2)OCO3)CC1</smiles><smiles>COc1cc(C(C#N)N2CCOCC2)cc(OC)c1OC</smiles><smiles>COc1cc(C(C#N)N2CCN(C)CC2)cc(OC)c1OC</smiles>

como control negativo se usó acetona, y disolvente de las diferentes concentraciones.

\section{Evaluación insecticida por aplicación tópica}

DE fueron aplicadas con microjeringa Hamilton de $5 \mu \mathrm{L}$ provista de descargador repetitivo. La aplicación de los tratamientos se realizó sobre los terguitos 
(región dorsal del abdomen del insecto) de cada ninfa en las cuales se aplicó $0,1 \mu \mathrm{L}$ de cada molécula a una dosis de 50, 12,5 o 2,5 ng/i. Todas las dosis fueron replicadas cuatro veces, con doce insectos por réplica y el experimento se realizó tres veces en días diferentes registrando la mortalidad desde 24 hasta las 72 horas pos tratamiento ${ }^{13,17}$.

\section{Evaluación insecticida por exposición de superficies tratadas}

Para estos experimentos se tomó como referencia el protocolo de la WHO (1996) con modificaciones ${ }^{13,18}$. Se usaron placas de Petri de vidrio de $6 \mathrm{~cm}$ de diámetro para la evaluación de superficies no porosas y para las evaluaciones de superficies porosas las mismas cajas fueron acondicionadas con discos de papel filtro (Munktell). Cada una de las placas se impregnó de manera homogénea con cada DE utilizando una micropipeta siguiendo un patrón en forma de espiral orientado del centro hacia afuera. El volumen utilizado fue de $437 \mu \mathrm{L}$ de la solución, la cual fue calculada según el protocolo WHO (1996). Después del secado de las superficies impregnadas se colocaron las ninfas, posteriormente se cubrieron las placas Petri con vinipel perforado y se tomó el registro de la mortalidad a las 24, 48, hasta las 72 horas pos tratamiento. Como criterio de muerte se estableció que el insecto no debía presentar actividad locomotora propia, ya sea de forma espontánea o cuando era estimulado con una pinza o pincel.

\section{Determinación de Dosis Letales (DL)}

Para realizar estos experimentos, se tomó la molécula que presentó mayor mortalidad con las DE. Luego de conocer el análogo con mayor porcentaje de mortalidad se realizaron nuevas evaluaciones empleando una nueva batería de dosis múltiples y asimétricas para poder determinar sus dosis letales. Las dosis utilizadas fueron: 200, 140, 100, 80, 50 ng/i, se utilizó deltametrina como control positivo, la cual a una concentración de $50 \mathrm{ng} / \mathrm{i}$ presenta una mortalidad del $100 \%{ }^{19}$, acetona como control negativo y disolvente de las diferentes concentraciones. Se realizó el mismo proceso experimental del tratamiento de topicación utilizado para las DE.

\section{Análisis actividad locomotora}

Para esta prueba se realizaron dos tipos de tratamiento, uno de aplicación tópica y otro de impregnación de superficies, para ambos tratamientos se tomaron tres ninfas de primer estadio para los experimentos. Sobre un papel filtro (Munktell) circular de 12,5 cm de diámetro se colocó un anillo de acrílico ( $4 \mathrm{~cm}$ de diámetro y 2 $\mathrm{cm}$ de alto) y posteriormente se introdujeron las ninfas y se cubrieron con papel vinipel para evitar que los individuos contenidos en el interior permanecieran el área del experimento. Se utilizó la $\mathrm{DL}_{50}$ de la molécula (6) que presentó la mayor mortalidad en los experimentos de topicación 1050,93 $\pm 1,53$ y deltametrina para $T$. dimidiata correspondiente a 5,02 $\pm 0,055 \mathrm{ng} / \mathrm{i}$ como control positivo y agua y acetona como controles negativos. Se emplean 12 ninfas por repetición por bioensayo y agua como segundo control negativo debido a que la volatilidad de la acetona podría llevar a suponer un estimulo en el movimiento de los insectos.

En la cuantificación de la actividad locomotora se registraron datos de movimiento pixel área con ayuda de una videocámara (VC 1910, Sanyo) adaptada en área experimental que crea un circuito cerrado de contraste blanco y negro. Se consideró como movimiento en el experimento la sumatoria de cambio de los pixeles de blanco a negro y viceversa en un periodo de tiempo de 30 minutos. Cada tratamiento y cada bioensayo fue replicado tres veces en diferentes días. Los registros de esta prueba fueron analizados en un procesador de imágenes Videomex $\mathrm{V}$ (Columbus $\mathrm{OH}$ ) de acuerdo a la metodología de Moretti, et al. 201720,21.

\section{Análisis estadísticos}

Los tratamientos fueron analizados de acuerdo con los tiempos de mortalidad (24, 48, 72 horas). Para todos los datos se realizaron prueba de normalidad y homocedasticidad (Kolmogorov Smirnov y Levene), posteriormente se realizó el análisis para datos que no tienen distribución no normal mediante la prueba no paramétrico Kruskall-Wallis (KW) o ANOVA de una vía según la normalidad de los datos. Se corroboraron las diferencias significativas entre los distintos tratamientos utilizando el software "Statistics V13". Para determinar las $\mathrm{DL}_{50} \mathrm{y} \mathrm{DL}_{95}$ se utilizó un análisis probit, mediante el software Polo Plus. ${ }^{22}$

\section{Consideraciones éticas}

Este trabajo fue respaldado por el comité de ética de acuerdo con CEINCI-UIS, acta $N^{\circ} 03 / 26$ de julio del 2013. 


\section{Resultados}

\section{Topicación dosis exploratorias (24 h)}

De las 12 moléculas utilizadas en las DE, la actividad insecticida fue observada en las moléculas 4, 6 y 9 en una DE de $50 \mathrm{ng} / \mathrm{i}$, donde la molécula 9 presentó mayor mortalidad $(8,3 \%)$. Sin embargo, se observó una baja mortalidad en esta dosis $(4,17-8,3 \%)$ y en las demás DE no presentó actividad ( $0 \%$ ). Las moléculas evaluadas fueron significativamente diferentes cuando se comparó con los controles (Kruskal-Wallis: H (4, $\mathrm{N}=40)=29,61111 \mathrm{P}<0,0000)($ Tabla 1).

Tabla 1. Mortalidad en ninfas del primer estadio de $T$. dimidiata, a las 24, 48 y 72 horas con una concentración de 50 ng/i en los bioensayos de topicación

\begin{tabular}{cccc}
\hline Moléculas & $\begin{array}{c}\text { Mortalidad } \\
(\% \pm \mathbf{D E}) \mathbf{2 4 h}\end{array}$ & $\begin{array}{c}\text { Mortalidad } \\
(\mathbf{\%} \pm \mathbf{D E}) \mathbf{4 8 h}\end{array}$ & $\begin{array}{c}\text { Mortalidad } \\
(\mathbf{\%} \pm \mathbf{D E}) \mathbf{7 2 h}\end{array}$ \\
\hline 1 & $0 \pm 0^{\mathrm{a}}$ & $4,17 \pm 0,70^{\mathrm{a}}$ & $8,33 \pm 0^{\mathrm{a}}$ \\
6 & $4,17 \pm 0,71^{\mathrm{a}}$ & $8,33 \pm 0^{\mathrm{a}}$ & $20,83 \pm 0,71^{\mathrm{ab}}$ \\
7 & $0 \pm 0^{\mathrm{a}}$ & $0 \pm 0^{\mathrm{a}}$ & $12,50 \pm 2,12^{\mathrm{a}}$ \\
9 & $8,33 \pm 1,41^{\mathrm{a}}$ & $12,50 \pm 0,70^{\mathrm{a}}$ & $16,66 \pm 1,41^{\mathrm{a}}$ \\
4 & $4,17 \pm 0,71^{\mathrm{a}}$ & $8,33 \pm 1,40^{\mathrm{a}}$ & $8,33 \pm 1,41^{\mathrm{a}}$ \\
Deltametrina (+) & $100,00 \pm 0^{\mathrm{b}}$ & $100,00 \pm 0^{\mathrm{b}}$ & $100,00 \pm 0^{\mathrm{b}}$ \\
Control (-) & $0 \pm 0^{\mathrm{a}}$ & $0 \pm 0^{\mathrm{a}}$ & $0 \pm 0^{\mathrm{a}}$ \\
\hline
\end{tabular}

En cada columna, letras iguales muestran que no hay diferencias estadísticamente significativas. $\mathrm{P}<0,05$ [KruskalWallis: H $(4, \mathrm{~N}=40)=29,61111 \mathrm{P}<0,0000] 24 \mathrm{~h}$, [KruskalWallis: $\mathrm{H}(5, \mathrm{~N}=48)=30,10938 \mathrm{P}<0,0000]$ [Kruskal-Wallis: $\mathrm{H}(6, \mathrm{~N}=56)=30.20974 \mathrm{P}<0,0000]$ 72h. DE: Desvio estandar.

\section{Topicación dosis exploratorias (48 h)}

La actividad insecticida fue observada para las moléculas 1, 4, 6 y 9 a una concentración de $500 \mathrm{ng} / \mathrm{i}$. Igualmente, la actividad insecticida fue baja en esta concentración $(4,17-12,5 \%)$ e inferior en las demás concentraciones $(>4,17 \%)$. La molécula 9 presentó mayor mortalidad con un valor del $12.5 \%$, sin embargo, se observó un aumento en la mortalidad al compararse con la obtenida a las 24 horas $(8,3 \%)$. Los resultados de esta prueba presentaron diferencias estadísticas significativas con respecto a los controles [KruskalWallis:H $(5, \mathrm{~N}=48)=30,10938 \mathrm{P}<0,0000]$ (Tabla 1).

\section{Topicación dosis exploratorias (72 h)}

Como resultado final solo cinco moléculas presentaron actividad insecticida, 1, 4, 6, 7 y 9, las cuales presentaron diferencias significatívas, [Kruskal-Wallis: H $\quad(6$,
$\mathrm{N}=56)=30.20974 \mathrm{P}<0,0000]$. La molécula que presentó mayor porcentaje de mortalidad fue el análogo $6(20,8 \%)$ seguido del análogo $9(16,6 \%)$, considerándose 6 como molécula más activa de las 12 evaluadas sobre ninfas del primer estadio de $T$. dimidiata (Tabla 1), además de ser la única en la que se observó actividad insecticida en el tratamiento de superficies.

\section{Exposición a superficies tratadas (DE)}

Aunque la molécula 6 presentó la mayor actividad insecticida, solo se observó mortalidad en su evaluación sobre la superficie no porosa. El control positivo deltametrina en el tratamiento de superficie porosa, presentó menor actividad insecticida con respecto al tratamiento de superficie no porosa. Se reporta la mortalidad luego de 72 horas con diferencias estadísticas significativas según ANOVA de una vía y el test de Tukey (F 3, $4=89$ p=0,004), (Tabla 2). Los tratamientos a las $24 \mathrm{~h}$ y $48 \mathrm{~h}$ no se tuvieron en cuenta para el análisis al no observarse diferencias.

Tabla 2. Mortalidad en el tratamiento de aplicación sobre superficies (porosa, no porosa) en ninfas del primer estadio de $T$. dimidiata en los distintos tratamientos, pasadas las 72 horas.

\begin{tabular}{ccc}
\hline $\begin{array}{c}\text { Moléculas } \\
\text { (Superficie) }\end{array}$ & Promedio & $\begin{array}{c}\text { Mortalidad } \\
(\% \pm D E)\end{array}$ \\
\hline 6 Porosa & $0^{\mathrm{a}}$ & $0 \pm 0$ \\
6 No Porosa & $3^{\mathrm{b}}$ & $25,0 \pm 0$ \\
Deltametrina Porosa & $9^{\mathrm{b}}$ & $75,0 \pm 1,4$ \\
Deltametrina no Porosa & $11,5^{\mathrm{c}}$ & $95,8 \pm 0,7$ \\
\hline
\end{tabular}

Letras iguales indican que no hay diferencias estadísticamente significativas. $\mathrm{P}<0,05$ (F 3, $4=89 \mathrm{p}=0,004)$. DE: Desvio estandar.

\section{Dosis múltiples y análisis probit}

Dada la baja mortalidad que se observó en las DE, se aumentaron las dosis aplicadas del análogo 6 (molécula evaluada con mayor actividad) para dilucidar las $\mathrm{DL}_{50}$ y $\mathrm{DL}_{95}$. Las dosis utilizadas fueron: 200, 140, 100, $80,50 \mathrm{ng} / \mathrm{i}$, respectivamente, determinando a las 72 horas sus valores $\mathrm{DL}_{50}=105,092 \pm 1,530 \mathrm{ng} / \mathrm{i} \mathrm{y} \mathrm{DL}_{95}=$ $415,01 \pm 0,508 \mathrm{ng} / \mathrm{i}$, respectivamente (Tabla 3).

\section{Análisis de la actividad locomotora}

En este análisis se realizó una comparación entre los dos tratamientos, topicación e impregnación de superficies, tomando como referencia las $\mathrm{DL}_{50}(1050,93 \mathrm{ng} / \mathrm{i}$ para 6 y $5,02 \pm 0,055 \mathrm{ng} / \mathrm{i}$ para deltametrina) y empleando 
como controles negativo acetona y agua (Tabla 3 ). Se logró observar mayor movimiento en el tratamiento por impregnación de superficies, con respecto al observado en el tratamiento de topicación. En la impregnación de superficies con la molécula 6 y deltametrina mostraron un mayor movimiento en las ninfas que el control negativo agua [ANOVA: $\mathrm{DF}=7, \mathrm{~F}=25,54$ ) $\mathrm{p}=0,000072]$. Sin embargo, las dosis aplicadas $\mathrm{DL}_{50}$ del control positivo deltametrina y el análogo 6 presentan un comportamiento similar en los dos tratamientos evaluados (topicación e impregnación), respecto a los controles negativos (Tabla 4).
Tabla 3. Dosis letaes DL ${ }_{50} \mathrm{DL}_{95}$ para la mejor molécula (analogo 6) en ninfas del primer estadio de T. dimidiata, a las 72 horas y el control positivo (deltametrina).

\begin{tabular}{cccc}
\hline Molécula & $\begin{array}{c}\mathbf{D L}_{\mathbf{5 0}} \\
{[\mathrm{ng} / \mathrm{i}]}\end{array}$ & $\begin{array}{c}\mathbf{D L}_{\mathbf{9 5}} \\
{[\mathrm{ng} / \mathrm{i}]}\end{array}$ & $\mathbf{X}^{\mathbf{2}}$ \\
\hline $\mathbf{6}$ & $1050,93 \pm 1,53$ & $4150,1 \pm 0,51$ & 1,282 \\
Deltametrina & $5,02 \pm 0,055$ & $20,163 \pm 0.56$ & 0,121 \\
\hline
\end{tabular}

DL50 = dosis que ocasiona el 50\% de mortalidad de los insectos expuestos, expresada en ng; DL95 = dosis que ocasiona el 95\% de la mortalidad de los insectos expuestos, expresada en ng/i. Las $\mathrm{DL}_{50}, \mathrm{y}$ $\mathrm{DL}_{95}$ para el insecticidad deltametrina. ${ }^{19}$

Tabla 4. Movimiento en las ninfas del primer estadio de Triatoma dimidiata representado como movimiento sobre área para la molécula 6 en los tipos de tratamiento (Impregnación y topicación) 30 minutos de grabación utilizando el equipo Videomex V (Columbos $\mathrm{OH})$.

\begin{tabular}{cccccc}
\hline Tipo de Tratamiento & Moléculas & Mínimo Movimiento & Máximo Movimiento & Promedio & $\begin{array}{c}\text { Desviación } \\
\text { Estándar }\end{array}$ \\
\hline \multirow{2}{*}{ Impregnación $^{*}$} & 6 & 1015,28 & 3374,78 & $2039,40^{\mathrm{a}}$ & 1210,15 \\
& Delta & 2636,72 & 3835,49 & $3386,31^{\mathrm{b}}$ & 653,40 \\
& Agua & 275,72 & 1514,47 & $1264,69^{\mathrm{a}}$ & 275,71 \\
& Acetona & 879,16 & 3780,51 & $2766,41^{\text {ac }}$ & 879,16 \\
Topicación $^{* *}$ & 6 & 549,28 & 2407,11 & $1855,57^{\mathrm{a}}$ & 549,28 \\
& Delta & 168,13 & 2174,89 & $2050,37^{\mathrm{b}}$ & 168,13 \\
& Agua & 683,65 & 2958,91 & $2176,06^{\mathrm{a}}$ & 683,65 \\
& Acetona & 1295,33 & 3332,78 & $2578,60^{\mathrm{bc}}$ & 1295,33 \\
\hline
\end{tabular}

Delta $=$ Deltrametrina.

Promedio: sumatoria del total de los registros de movimiento dividido por el número de réplicas.

*Indican diferencias significativas entre los tratamientos $P<0,05$, [ANOVA: $\mathrm{DF}=3, \mathrm{~F}=23,100) \mathrm{p}=0,00027]$.

Letras iguales muestran que no hay diferencias estadísticamente significativas. $P<0,05$, [ANOVA: $\mathrm{DF}=7, \mathrm{~F}=25,54) \mathrm{p}=0,000072$ ].

\section{Discusión}

\section{Acción de los análogos evaluados}

Los compuestos sintéticos evaluados en este trabajo son de naturaleza hidrofóbica por lo que presentan baja solubilidad en sistemas acuosos y alta en disolventes orgánicos como la acetona. Además, estudios preliminares demostraron que los compuestos evaluados no son volátiles bajo las condiciones de evaluación. Estas características indican que al disolver los diferentes análogos en acetona y topicarlos sobre la ninfa, es de esperar que el proceso de evaporación del disolvente lleve a la cristalización de los sistemas en la superficie del insecto. Este estado de agregación dificulta la permeabilidad de los análogos a través de la cutícula generando baja toxicidad en el insecto, efecto que se acentúa cuando se evalúan estadios superiores al primero. Este efecto fue reportado por Cuadros et al, ${ }^{13}$ razón por la cuál las ninfas de $R$. prolixus de primer estadio fueron más susceptibles que las de quinto estadio a causa de la cantidad de molécula que logra entrar al insecto. Este análisis se hace en comparación con el principal insecticida (piretroide) usando actualmente contra los vectores de la enfermedad de Chagas ${ }^{23}$.

La determinación de los valores de $\log P$ (coeficiente de reparto octanol/agua), como indicadores de la lipofilicidad de las moléculas, mostró que entre los sistemas evaluados con el fragmento pirrolidínico, la molécula $6(\operatorname{LogP} 1,97)$ presenta un carácter lipofílico mayor, lo que le permitiría permear más fácilmente la cutícula cerosa de los triatominos. Este resultado es coherente con el observado por Cuadros, et al. ya que en la especie $R$. prolixus esta molécula presentó resultados altos en ninfas de primer estadio y bajos cuando se evaluó sobre el quinto estadio, instar donde los insectos de esta especie presentan una cutícula más desarrollada ${ }^{13}$. Aunque la evaluación de las moléculas sobre dos especies de triatominos demostró que los 
análogos 3 y 6 son los de mayor acción insecticida sobre ninfas de $R$. prolixus y $T$. dimidiata, respectivamente, su equivalencia estructural y características fisicoquímicas semejantes permitió determinar que la especie $R$. prolixus es mas susceptible a la acción de los análogos evaluados que ninfas de $T$. dimidiata.

En los resultados, el control deltametrina siempre presentó una mortalidad del $100 \%$ a una concentración de $500 \mathrm{ng} / \mathrm{i}$, en comparación con la mejor molécula 6 que a esta misma concentración solo presenta una mortalidad del $20,83 \% \pm 3,57$. Esta diferencia de mortalidad puede ser explicada por las fases tóxico cinéticas y tóxico dinámicas, los cuales son procesos que se presentan a medida que un insecticida ingresa e interacciona con diferentes sistemas del insecto ${ }^{24}$.

Finalmente, la diferencia en la actividad insecticida entre la molécula 6 y la cipermetrina podría explicarse debido a que su objetivo molecular no es alcanzado con eficiencia a causa de la baja absorción por la cutícula del insecto. Además, en Carreño-Otero, et al. ${ }^{14}$ se argumentó que los análogos de girgensohnina inhibían la AChE, indicando así el mecanismo de acción insecticida de esta molécula. Sin embargo, en un estudio reciente de Borrero, et al. ${ }^{25}$ se ha demostrado que estos compuestos también afectan la respiración celular inhibiendo el transporte de electrones en la mitocondria, lo cual podría resultar en un proceso de mortalidad más lento que cuando se compara con insecticidas que afectan el sistema nervioso de los insectos como los piretroides. Por otro lado, es evidente que ninfas de estadios avanzados son menos susceptibles a los insecticidas como se observa en este estudio. Este mismo resultado fue observado previamente en ensayos de toxicidad con deltametrina evaluados sobre diferentes estadios de desarrollo de T. infestans, mostrando que las ninfas del primer estadio son más susceptibles. ${ }^{26}$

\section{Superficies}

En el bioensayo de superficies, la molécula 6 presentó acción insecticida en el tratamiento no poroso. Este resultado posiblemente tenga la misma explicación que el experimento de topicación donde hay una baja absorción de la molécula a la cuál se expusieron las ninfas. Los análogos de girgensohnina son moléculas no volátiles que para su impregnación son disueltas en acetona, disolvente altamente volátil como se explicó anteriormente. Debido a esta característica se pueden formar cristales, tanto en las moléculas evaluadas como en el control positivo de acetona, las cuales se adhieren al papel filtro internalizándose en sus porosidades disminuyendo el contacto con el insecto. A diferencia de la superficie no porosa que corresponde a la base de vidrio de la caja Petri, en la cual el total de la sustancia se deposita sobre la superficie y hace que esté en contacto con los tarsos de los insectos. Razón por la cual el tratamiento de superficie no porosa presenta una mayor acción insecticida tanto con las moléculas como con el control positivo ${ }^{27}$. Estudios de campo corroboran esta afirmación con diferentes insecticidas, en las cuales las superficies porosas presentan una menor efectividad con respecto a superficies no porosas ladrillo y concreto ${ }^{28}$.

\section{Locomoción}

En el bioensayo de locomoción se comparan dos tratamientos, topicación e impregnación sobre superficie, esto debido al bajo resultado observado en el bioensayo de exposición a superficies, de igual manera se evaluó un control negativo adicional debido a la volatilidad que posee la acetona, razón por la cual se empleó agua destilada como segundo control negativo. La hiperactividad en los insectos es uno de los primeros síntomas de intoxicación producido por los piretroides ${ }^{29}$. En el tratamiento de impregnación de superficies se observó que el control positivo (deltametrina) indujo un movimiento mayor al compararlo con el análogo 6. La exposición de las ninfas a estas dos moléculas mostró un mayor movimiento que el control negativo (agua), este fenómeno es debido a la fase de excitación prolongada que sufre el insecto expuesto en la superficie. Sin embargo, en el tratamiento de topicación, tanto la mejor molécula como el control positivo, muestran valores de movimiento menores a los controles negativos. Este fenómeno puede deberse a que el insecto entra en una fase de tetanización, caracterizada por movimientos convulsivos y violentos en todas sus patas ${ }^{21}$, razón por la cual su movimiento se ve impedido por la acción del insecticida. Dado que al aplicarse directamente las moléculas sobre el dorso del individuo los sistemas evaluados pueden actuar de una manera más rápida, su fase de excitación no es tan prolongada como en el tratamiento de impregnación a superficies. Esto indica que a pesar de que las moléculas evaluadas (análogo 6 y deltametrina) tienen diferentes mecanismos de acción e incluso son evaluadas a dosis diferentes, estas moléculas llevan a inducir un comportamiento similar. De cualquier forma la hiperactividad producida por los piretroides en tratamientos de impregnación en triatominos, y la sobre estimulación del sistema nervioso por parte de inhibidores de la AChE ya se han confirmado en estudios anteriores ${ }^{30}$. 


\section{Agradecimientos}

Departamento Administrativo de Ciencia, Tecnología e Innovación de Colombia, COLCIENCIAS and Patrimonio Autónomo, Fondo Nacional de Financiamiento para la Ciencia, Francisco José de Caldas", código de contrato 110265740528 (convocatoria 624-2014).

\section{Referencias}

1. Rassi A Jr, Rassi A, Marcondes de Rezende J. American trypanosomiasis (Chagas Disease). Infect Dis Clin North Am. 2012; 26(2): 275-291. doi: 10.1016/j.idc.2012.03.002.

2. Sales Junior PA, Carneiro CM, Sánchez-Montalvá A, de Oliveira RC, Fonseca Murta SM, Salvador F. Experimental and clinical treatment of chagas disease: a review. Am J Trop Med Hyg. 2017; 97(5): 1289-1303. doi: 10.4269/ajtmh.16-0761.

3. Reisenman CE, Lawrence G, Guerenstein PG, Gregory T, Dotson E, Hildebrand JG. Infection of kissing bugs with trypanosoma cruzi, Tucson, Arizona, USA. Emerg Infect Dis. 2010; 16(3): 400405. doi: 10.3201/eid1603.090648.

4. Boiani M, Piacenza L, Hernández P, Boiani L, Cerecetto H, González M, et al. Mode of action of Nifurtimox and N-oxide-containing heterocycles against Trypanosoma cruzi: Is oxidative stress involved? Biochem Pharmacol. 2010; 79(12): 17361745. doi: 10.1016/j.bcp.2010.02.009.

5. Reyes-Novelo E, Ruiz-Piña HA, EscobedoOrtegón J, Barrera-Pérez MA. Biología y ecología de Triatoma dimidiata (Latreille, 1811), algunos aspectos de estudio. Dugesiana. 2011;18(1):11-16.

6. Justi SA, Galvão C. The evolutionary origin of diversity in Chagas disease vectors. Trends Parasitol. 2017; 33(1): 42-52. doi: 10.1016/j.pt.2016.11.002.

7. Alzogaray R A. El control químico de Triatoma infestans en Argentina. Retel - Rev Toxicol Lin. 2003; 1: 1-18.

8. Guhl F, Aguilera G, Pinto NA, Vergara D. Actualización de la distribución geográfica y ecoepidemiología de la fauna de triatominos (Reduviidae: Triatominae) en Colombia. Biomédica. 2007; 27(1): 143-162.

9. Palomino M, Villaseca P, Cárdenas F, J A, Pinto M. Eficacia y residualidad de dos insecticidas piretroides contra Triatoma infestans en tres tipos de viviendas. evaluación de campo en Arequipa, Perú. Rev Peru Med Exp Salud Publica. 2008; 25(1): 9-16. 10. Carneiro ÂP, Josene Barbosa Pereira M, Galbiati C. Biocide activity of Annona coriacea seeds extract on
Rhodnius neglectus (Hemiptera: Reduviidae). Rev Biol Trop. 2013; 61(1): 419-427.

11. Mougabure-Cueto G, Picollo MI. Insecticide resistance in vector Chagas disease: Evolution, mechanisms and management. Acta Trop. 2015; 149: 70-85. doi: 10.1016/j.actatropica.2015.05.014.

12. Vargas LY, Kouznetsov VV. First Girgensohnine Analogs Prepared Through $\mathrm{InCl}_{3}$-catalyzed Strecker Reaction and their Bioprospection. Curr Org Synth. 2013; 10: 969-973.

13. Cuadros J, Carreño Otero A, Kouznetsov V, Duque JE. Insecticidal action of synthetic girgensohnine analogs and essential oils on Rhodnius prolixus (Hemiptera: Reduviidae). Biomed - Rev Inst Nac Salud. 2017; 37(2): 1-27.

14. Carreño Otero AL, Vargas Méndez LY, Duque L JE, Kouznetsov VV. Design, synthesis, acetylcholinesterase inhibition and larvicidal activity of girgensohnine analogs on Aedes aegypti, vector of dengue fever. Eur J Med Chem. 2014; 78: 392-400. doi: 10.1016/j.ejmech.2014.03.067.

15. Alzogaray, R. A. Behavioral and toxicological responses of Rhodnius prolixus (Hemiptera: Reduviidae) to the insect repellents DEET and IR3535. J Med Entomol. 2016; 53: 387-393. doi: 10.1093/jme/tjv194.

16. Reyes M, Angulo VM. Ciclo de vida de Triatoma dimidiata Latreille, 1811 ( Hemiptera, Reduviidae ) en condiciones de laboratorio : producción de ninfas para ensayos biológicos. Biomedica. 2009; 1811: 119-126.

17. Robertson JL, Savin NE, Preisler HK, Russell RM. Bioassays with Arthropods, Second Ed. 2007; 102, 224.

18. World Health Organization - WHO. Report of the WHO informal consultation on the evaluation and testing of insecticides. 1996; 76.

19. Reyes M, Angulo VM, Sandoval CM. Efecto tóxico de $\beta$-cipermetrina, deltametrina y fenitrotión en cepas de Triatoma dimidiata (Latreille , 1811) y Triatoma maculata (Erichson, 1848) (Hemiptera, Reduviidae). Biomedica. 2007; 27(Supl. 1): 75-82.

20. Moretti AN, Seccacini EA, Zerba EN, Canale D, Alzogaray RA. The botanical monoterpenes linalool and eugenol flush-out nymphs of Triatoma infestans (Hemiptera: Reduviidae). J Med Entomol 2017; 54: 1293-1298.

21. Alzogaray RA, Fontán A, Zerba EN. Evaluation of hyperactivity produced by pyrethroid treatment on third instar nymphs of Triatoma infestans (Hemiptera:Reduviidae). Arch Insect Biochem Physiol. 1997; 35(3): 323-333. doi: 0.1002/ (SICI)1520-6327(199705)35:3<323::AID- 
ARCH6 $>3.0 . \mathrm{CO} ; 2-\mathrm{U}$

22. PoloPc. POLO-Plus, POLO for Windows computer program, version 2.0. LeOra-Software, Petaluma, CA. 2007.

23. Sfara V, Zerba EN, Alzogaray RA. Toxicity of pyrethroids and repellency of diethyltoluamide in two deltamethrin-resistant colonies of Triatoma infestans Klug, 1834 (Hemiptera: Reduviidae). Mem Inst Oswaldo Cruz. 2006; 101(1): 89-94. doi: 10.1590/S0074-02762006000100017.

24. Carreño Otero AL, Palacio-Cortés AM, NavarroSilva MA, Kouznetsov VV, Duque L. JE. Behavior of detoxifying enzymes of Aedes aegypti exposed to girgensohnine alkaloid analog and Cymbopogon flexuosus essential oil. Comp Biochem Physiol C Toxicol Pharmacol. 2018; 204: 14-25. doi: 10.1016/j.cbpc.2017.11.002.

25. Borrero Landazabal MA, Carreño Otero AL, Kouznetsov VV, Duque Luna JE, Mendez-Sanchez SC. Alterations of mitochondrial electron transport chain and oxidative stress induced by alkaloid-like $\alpha$-aminonitriles on Aedes aegypti larvae. Pestic Biochem Physiol. 2018; 144: 64-70. doi: 10.1016/j. pestbp.2017.11.006.

26. Germano, M. D. Herencia y efectos demográficos de la resistencia a deltametrina en Triatoma infestans. Centro de Investigaciones de Plagas e Insecticidas. Buenos Aires, 2012; Tesis Doctoral.

27. Stampini M, Girgenti P, Locatelli DP. Activity of Deltamethrin on different surfaces against Plodia interpunctella larvae (Lepidoptera Pyralidae). Ist Entomol Agrar Univ Stud Milano. 2008; 1-6.

28. Palomino M, León W, Valencia P, Cárdenas F, Ancca J. Evaluación de campo del efecto residual de la Deltametrina sobre la mortalidad y knockdown en Triatoma infestans, según tipo de supeficie en Arequipa, Perú. Rev Per Med Exp Salud Publica. 2007; 24(2): 136-143.

29. Alzogaray R, Sfara V, Moretti AN, Zerba EN. Behavioural and toxicological responses of Blattella germanica (Dictyoptera: Blattellidae) to monoterpenes. Eur J Entomol. 2013; 110(2): 247-252.

30. Xuereb B, Lefèvre E, Garric J, Geffard O. Acetylcholinesterase activity in Gammarus fossarum (Crustacea Amphipoda): Linking AChE inhibition and behavioural alteration. Aquat Toxicol. 2009; 94(2): 114-122. doi: 10.1016/j. aquatox.2009.06.010. 